\title{
Recognition of Hand-Printed Chinese Characters Using Ripple Down Rules
}

\author{
Adnan Amin, Michael Bamford, Achim Hoffmann, Ashesh Mahidadia, and \\ Paul Compton
}

School of Computer Science and Engineering

University of New South Wales, 2052 Sydney, Australia

(Email: \{amin,bamford,achim,ashesh,compton\}@cse.unsw.edu.au)

\begin{abstract}
The paper presents a prototype knowledge acquisition system which has been implemented based on Ripple Down Rules. The main aim of our system is to capture the human skills of recognizing and differentiating a character from other similar characters. The user can identify such differences graphically and (if required) modify the Knowledge Base without the guidance of a Knowledge Engineer. The paper outlines the basic framework and presents initial experimental results. Further, it discusses ways of scaling up the approach to handle very large number of Chinese characters.
\end{abstract}

\section{Introduction}

Recognition of Chinese characters has been a major interest of researchers for many years, and a large number of research papers and reports have already been published in this area. There are several major problems: Chinese characters are distinct and ideographic, the character size is very large and a lot of structurally similar characters exist in the character set. Thus, classification criteria are difficult to find.

Research in Chinese character recognition faces many difficulties. First, there are a large number of Chinese characters (more than 50,000 characters, of which 6,000 are commonly used). Second, Chinese characters have a more complex structure than alphabetic characters and there are a large number of mutually similar characters. Many characters may have exactly the same structure except that the length of the strokes are different. These issues become more complicated in hand-printed Chinese character recognition where characters can appear in different fonts and sizes.

In view of these problems, various methods have been proposed in the past. These methods can be classified into two categories. One approach is stroke analysis that extract features such as endpoints, crossing points, stroke relative positions, etc from the input pattern. Another approach is pattern matching, which is based on the comparison between the input distribution function and the standard one.

Many researches tend to use the stroke analysis approach. For example, Augi [1] uses the concatenation relation, cross relation and near relation to analysis Chinese characters. Yamamoto [10] uses the stroke direction, stroke domain 
and stroke density to classify characters. Hsieh and Lee [5] use a one-dimensional string consisting of a stroke sequence interleaved with relationships between two consecutive strokes to represent a character.

The new approach targets at capturing human skills of recognising and differentiating a character from other similar characters. The user can identify such differences graphically and (if required) modify the knowledge base without the guidance of a knowledge engineer. Hence the advantages of the RDR methodology [3] is that the user can build and maintain the knowledge base efficiently without the guidance of a knowledge engineer. Also the need to analyse and recognise the knowledge in the knowledge base is eliminated. The new approach also makes it easy to modify the knowledge base to cover variations in writing styles. The paper describes the approach and initial experimental results. Figure 1 illustrates the new aproach to construct a Knowledge Base Classifier.

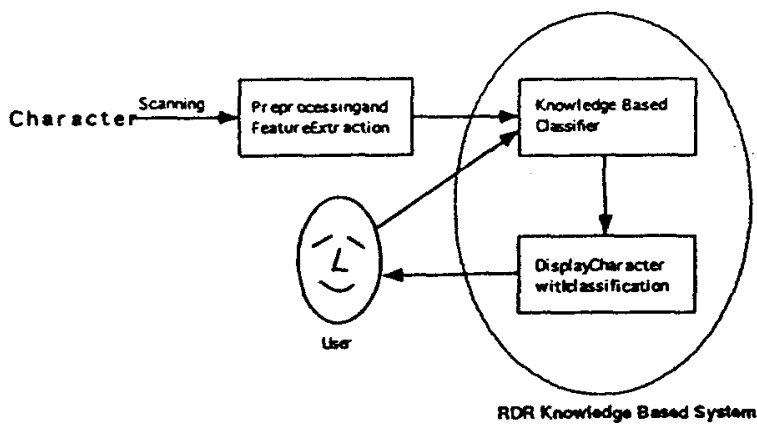

Fig. 1. The user checks the classification of the knowledge-based classifier. If the system's classification is incorrect, the user specifies additional conditions using graphical interface to update the knowledge base.

\section{Digitisation and Preprocessing}

\subsection{Digitisation}

The first phase in our character recognition system is digitisation. Hand printed Chinese characters are first scanned and digitised. A 300 dpi scanner is used in this step and the size of the characters is approximately $30 \mathrm{~mm} \times 30 \mathrm{~mm}$.

\subsection{Pre-thinning and Thinning}

This step aims to reduce the noise that the binarization process yields. The prethinning algorithm used in this paper is similar to that which appears in [7]. 
The thinning algorithm adopted in this paper is Jang and Chin's one pass parallel thinning algorithm [6] because it gives skeletons with few spurious branches. After thinning, the maximum circle technique proposed by Liao and Huang [7] is used to remove the spurious branches.

\section{$3 \quad$ Tracing}

An algorithm implementing a $3 \times 3$ window is used to trace along the path of the skeleton, recording the Freeman codes $(0 \mathrm{E}, 1 \mathrm{NE}, 2 \mathrm{~N}, 4 \mathrm{~W}, 5 \mathrm{SW}, 6 \mathrm{~S}, 7$ $\mathrm{SE}$ ) [4] along the path. The Freeman code simply attaches a number to each of the eight major points of the compass and the orientation of a line segment is characterized by this number. A path is described as a trace between junction or end points, where an end point has a single neighbour and a junction point has two neighbours. Whenever a junction point is reached, a depth-first search is used to follow each path in turn.

The end points in the preprocessed image are used as starting points for tracing. As we trace out the codes, we remove the pixel from the thinned image. After all the end points have been visited, the thinned image should only contain closed loops. We search this image for any remaining pixels, and use the first pixel encountered as our starting point.

The result of the tracing algorithm is a set of line segments. We then apply a stroke segmentation technique, using inner products of the line segments [2] to join the line segments into strokes. The result of the stroke segmentation is the input to our feature extraction block. An example of preprocessing is shown in Figure 2.

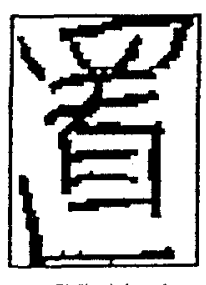

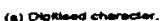

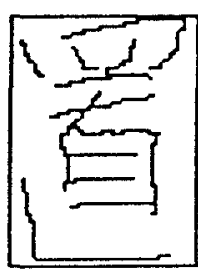

(o) Nerer monters

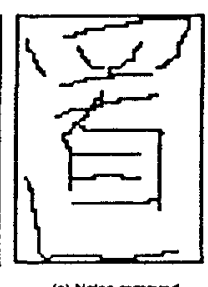

(a) Nolese nomones

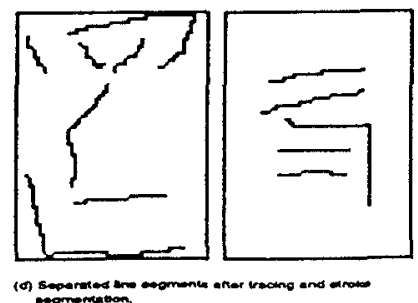

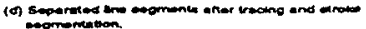

Fig. 2. Preparing input to feature extraction.

\section{Feature Extraction}

In this project we have used six primitives (see Figure 3) extracted by using the dominant point method. 


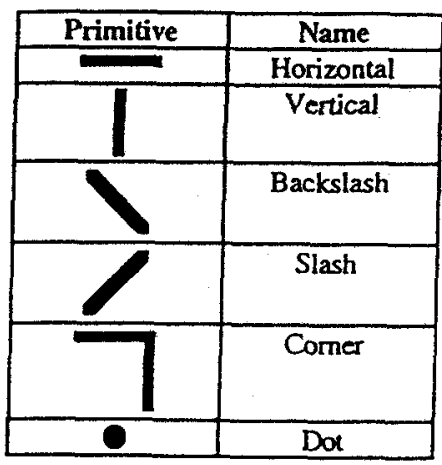

Fig. 3. Primitive features used in the system.

A number of dominant point detection algorithms have been proposed in the past. These algorithms have been summarized and compared by Teh and Chin [9]. However, in character recognition, the requirements of a good dominant point detection algorithm are slightly different from those of algorithm for general usage. In character recognition, we do not have to identify all dominant points. We only need to identify points with very sharp curvature.

Based on this requirement, we were inspired by the Rosenfeld-Johnston algorithm [8]. The basic concept of the algorithm is to calculate the curvature of each point in the line, then the point with local maximum in curvature are designated as the dominant points. However, the results were not satisfactory. Due to the irregularity of the lines, we detected too many dominant points in the line segments. For example, the line in Figure 4 should only be split into three parts (two vertical lines and one horizontal line). However, due to the irregularities at the middle of the horizontal line, the line was split into six parts.

A good smoothing algorithm can help in eliminating many of the undesirable dominant points. In addition, we need an algorithm to merge back some of the segments.

After analyzing the resuits of the dominant point extraction, we found that most of the undesirable dominant points were due to small irregularities of the chain code pattern. From this observation, we implemented a simple merging algorithm. The basic concept is that we try to merge segments that are really short with the adjacent long segments. Then we check the long segment. If there are two long segments of the same type, then we merge them together. The formal algorithm is described as follows:

Thus in Figure 4, line segments 3 and 4 are short line segments. Line segments 2 and 5 are long line segments and are of the same type (horizontal). Therefore segments $2,3,4$ and 5 will be merged together to form one long horizontal line. 

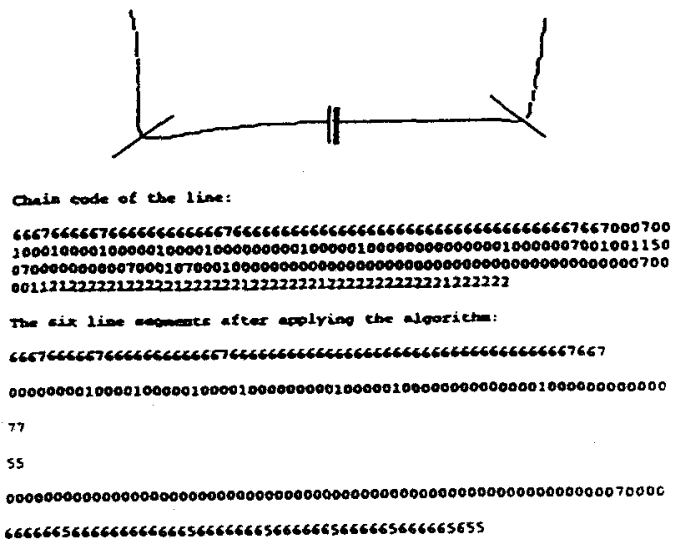

Fig. 4. The results of applying Dominant Point Algorithm. The line segment is split into 6 segments, rather than the 3 segments that we wanted.

Input: Line segments $L_{1}, L_{2}, \ldots, L_{n}$ which are the result of splitting an input line segment $L$ by dominant point method.

Output: Merged line segments $M_{1}, M_{2}, \ldots, M_{k}$, where $k \leq n$. Method:

1. We consider line segments with the line smaller than a threshold $t$, which is determined by experiment, as short line segments. Other line segments are long line segments.

2. If a short line segment is at the end of the original input line segment $L$, and its neighbour (the short line segment has only one neighbour) is a long line segment, then we merge these two line segments into one, and the type is considered as the same as the long line segment.

3. If a sequence of short line segments $L_{i}, \ldots, L_{j}$ lie between two long line segments $L_{i-1}$ and $L_{i+1}$, also $L_{i-1}$ and $L_{j+1}$ are of the same type, then we merge $L_{i-1}, L_{i}, \ldots, L_{j}, L_{j+1}$ into one line segment, and the type of the result is the same as $L_{i-1}$ and $L_{j+1}$.

Fig. 5. Algorithm: Re-merge Over-splitted Strokes

\section{Ripple Down Rules approach}

The essential idea of RDR is the following: An expert observes the performance of the KBS - in our case character classifier. If the system provides incorrect classification, the expert is presented with all the features exhibited by the case which were not considered by the system when determining its (incorrect) classification. The expert is then asked to identify the critical features from this list which may lead to the correct classification. Based on this, the system automati- 
cally updates the knowledge base. This provides substantial help to an expert to refine and correct the classification rules the system is currently using. Applied to character recognition, this means that the expert is merely asked to explain why he/she classifies a given character differently from the automatic classifier so far. By pointing out the differences between what the expert considers crucial and the system's classification rule being used, the automatic classifier is updated and refined step by step. To explicate differences with optical patterns like Chinese characters, a graphical interface is implemented which displays the current character and indicates those features which have led the automatic classifier to the incorrect classification. Using that information, an expert can easily recognise the reasons for the system's incorrect classification. By giving these reasons, e.g. the top horizontal stroke needs to be considered (see Figure 6), the refinement of the classification rules can be done.

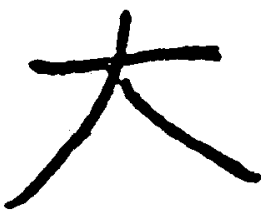

Big

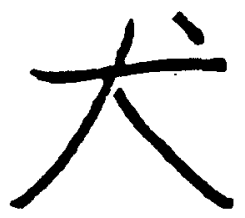

Dog

Fig. 6. Two structurally similar Chinese characters with different meanings. The current classifier may produce these two different possible classes for a given input. The user can disambiguate this to a single correct class by identifying presence of relevant strokes for each different character.

We also expect that the approach can be extended to further problems of Pattern Recognition and Computer Vision. Our initial studies of applying RDR for recognising Chinese characters show promising results. The following section briefly describes the new approach.

\section{The Interface for the RDR system}

The program supports a graphical user interface to enable the efficient selection of distinguishing strokes of a character. Usually, handwritten characters of a single class vary significantly in their appearance, e.g. with respect to the Freeman code, due to variations in writing styles etc. Using an intuitive graphical interface is an attempt to overcome this problem by allowing the expert to clearly 
distinguish those strokes that are least affected by variations in character writing and machine coding.

When an unknown character is presented to the application, the dialogue window as seen in Figure 7 (left) is presented. The character is presented using the machine codes that were generated in the feature extraction and tracing processes. The width of the stroke and the writer's start and end points of the stroke are unimportant and are not included in the machine coding. Each strokes start and end points are indicated by a small cross. Note that the start and end points may not coincide with a traditional writers perception of a stroke's start and end points.
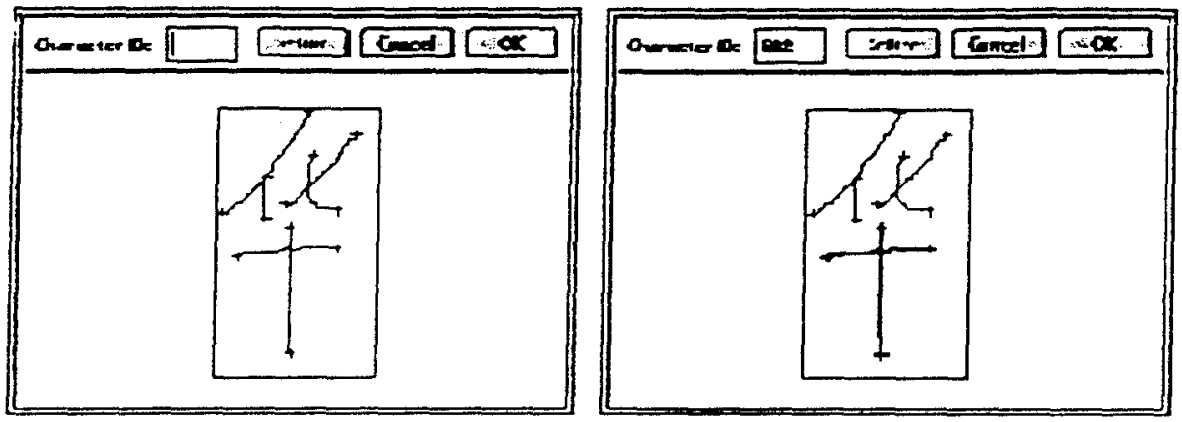

Fig. 7. Left: A Chinese character is displayed. Right: Two strokes are selected for recognizing the character.

The user is then able to select those strokes that distinguish the character and provide an identifier. In Figure 7 (right), the user has selected the two lower cross strokes. This provides a single rule for the RDR tree. Although the user is able to select any number of strokes, experimental results have shown that no more than three strokes should be selected at the early stages of building the expert system.

Subsequent samples of the same character may or may not be recognised according to the new rule added to the RDR rule. If the sample character's strokes satisfy the entered rule, the application will show the character with its identifier as illustrated in Figure 8 (left). The strokes that have satisfied rules in the RDR tree are displayed apart in hatch from other strokes.

Samples of the same character may not satisfy the rule because of different writing styles or variations during the feature extraction and as a consequence in the machine coding of the character. In these cases, the user will be required to select and enter additional rules to distinguish the new sample. Alternatively, it is possible that a different character is unrecognised because of coincidental strokes. In this case, the user will be required to again select strokes and enter additional rules to distinguish the character as illustrated below. In this figure, the bottom cross strokes have fired for a rule for character 'China' (602) have been highlighted (hatched) to indicate this. Subsequently, the user has clicked 

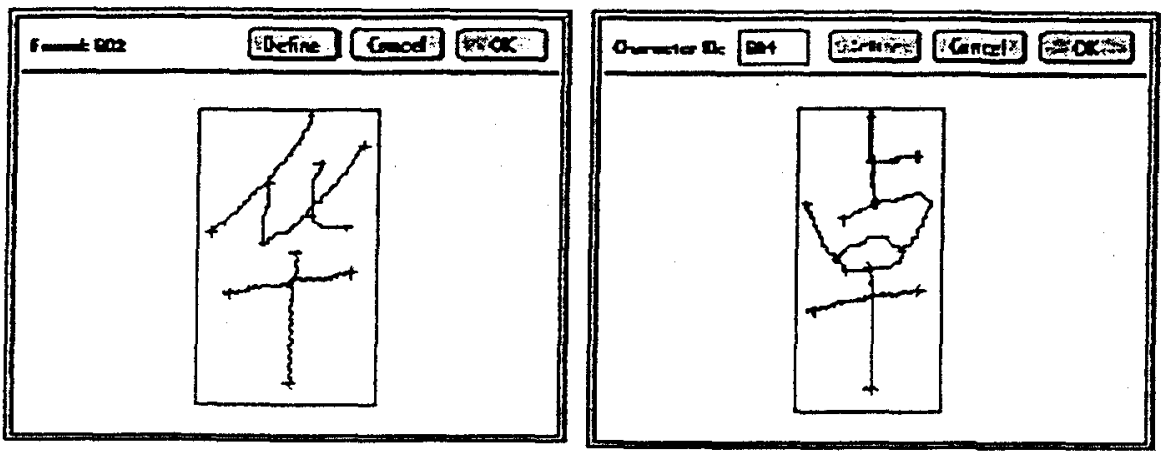

Fig. 8. Left: The character has been classified as 'China' (class 602). The classification is based on the two grey strokes. Right: The character has been misclassified based on the highlighted (hatched) strokes at the bottom. Additional strokes have been clicked on by the user to refine the classification rule for the class "outstanding' (class 604).

in the Define button to indicate that the character needs to be redefined since it has been misclassified. In figure 8 (right), the user has selected the top two strokes to distinguish the character.

The RDR expert tree stores both the rules of the stroke to distinguish the character as well as the machine coding representation of the character itself. When a misclassification occurs, the user must select distinguishing strokes that best represent the character by observing the following two conditions:

1. No strokes that have fired in rules that lead to the misclassification can be selected. Selecting such characters would not provide any newly distinguishing features to the character.

2. Strokes that exist in the new sample character as well as the character that provides the misclassification cannot be selected since doing so provide a correct classification for the new sample but the previously classified character would now be misclassified with the new sample.

In Figure 8 (right), the user cannot select the highlighted (hatched) strokes since these strokes have already been used in the classification processes.

\section{Matching the primitives in the new image}

One important issue in the development and application of RDR trees as described above is the following: A decision node in an RDR tree contains a condition which requires the presence or the absence of a specified primitive in the image to be classified. The primitive in the RDR tree's condition is specified by its type and by its start and end points.

The problem is to determine whether a primitive of the same type in a new image matches the condition or not. 
The current approach to that problem is very crude. First of all, we are considering the normalized image. An start or end point is considered to be matching the stroke represented in the RDR tree, if the respective points in the new image are matching these points. Let the (normalized) width of the normalized character be denoted by $w$ and the height by $h$ respectively.

Two points $p_{1}=\left(x_{1}, y_{1}\right)$ and $p_{2}=\left(x_{2}, y_{2}\right)$ are considered to match, if the distance $\left|x_{1}-x_{2}\right|$ is less than $\alpha \times w$ and if the distance $\left|y_{1}-y_{2}\right|$ is less than $\alpha \times h$. So far, we experimented with different values for $\alpha$ between $0 \%$ and $30 \%$. See also the final section on a discussion of the matching criteria.

\section{Experimental Results and Discussion}

The current implementation of the application is being developed in $\mathrm{C}++$ on Macintosh using Metrowerks PowerPlant framework. The most important aspect of the application is the RDR tree iterator and the stroke matching algorithm.

The iterator processes the tree, each node containing a rule, consisting of any number of strokes, and the machine coding of the sample character that generated the rule. Currently the data stored for each stroke includes the stroke's start and end points and its primitive type.

Experimental results have shown the current RDR system operates best when the user enters one to three strokes per rule. Too many strokes produces rules that are two specific that do not take into account variations in writing styles and feature extraction. Using a training set of 30 samples and a testing test of 10 samples, the average recognition was $96 \%$. However, as the number of character classes in the RDR tree increased, the number of rules that were required to distinguish the characters of different classes also increased. Table 1 illustrates this for $\alpha=0.2$. For an $\alpha$-value of $30 \%$ the classification of characters deterio-

\begin{tabular}{|l|c|l|}
\hline character classes & $\begin{array}{c}\text { Avg. number of strokes } \\
\text { per rule }\end{array}$ & Recognition rate \\
\hline First 50 & 8 & $92 \%$ \\
Second 50 & 15 & $96 \%$ \\
Third 50 & 23 & $96 \%$ \\
Fourth 50 & 30 & $96 \%$ \\
\hline
\end{tabular}

Table 1. Experimental results on recognizing Chinese characters. The matching criterion for the start and end points used an $\alpha=20 \%$.

rated quickly when more than 20 different classes were considered. This is to be expected since often primitives in the rules match primitives in the images to be classified where no matching is intended. For smaller $\alpha$-values more rules were required to have a similar classification accuracy. The increased number of rules had in particular the burden of asking the user to enter more and more rules 
manually. Therefore, our experiments so far used a relatively coarse $\alpha$-value of $20 \%$. However, at about 250 different classes the capacity of the coarse matching with $\alpha=0.2$ was exhausted and more and more ambiguities in the produced classification of our RDR rules occurred.

The current primitive matching approach has the disadvantage that it is fairly crude on the one hand, and a fixed predefined value for $\alpha$ is being used.

It would be desirable, if the user gets more control on what matching criteria is applied for each primitive individually which he/she specifies as a condition in the RDR rules.

Currently, we are investigating alternative matching criteria, such as allowing the user to specify individual areas for acceptable start and end points of primitives. Furthermore, additional primitives are considered to be included in the current collection. Also additional characteristics of a character will be included into our visual Knowledge Acquisition tool including the specification of the number of different types of primitives in a character, the relationships between radicals or groups of strokes.

\section{References}

1. T. Augi and H. Nagahaski. A description method of handprinted Chinese characters. IEEE Trans. PAMI, 1979.

2. F. Cheng and W. Hse. Three stroke extraction methods for recognition of handwritten chinese characters. In Proc. Int. Conf. Chinese Computing, pages 191-195, 1986.

3. P. Compton, G. Edwards, B. Kang, L. Lazarus, R. Malor, P. Preston, and A. Srinivasan. Ripple down rules: Turning knowledge acquisition into knowledge maintenance. Artificial Intelligence in Medicine, 4:463-475, 1992.

4. H. Freeman. On the encoding of arbitrary geometric configurations. IEEE Transactions on Electronic Computers EC-10, pages 260-268, 1968.

5. C. Hsieh and H. Lee. Off-line recognition of handwritten Chinese characters by online model-guided matching. Pattern Recognition, (25):1337-1352, 1992.

6. B. Jang and R. Chin. One-pass parallel thinning: Analysis, properties, and quantitative evaluation. IEEE Trans. Pattern Anal. Mach. Intell. PAMI-14, pages 1129-1140, 1992.

7. C. Liao and J. Huang. Stroke segmentation by bernstein-bezier curve fitting. Pattern Recognition, (23):475-484, 1990.

8. A. Rosenfeld and E. Johnston. Angle detection on digital curves. IEEE Transactions on Computers C-22, pages 875-878, 1973.

9. C. Teh and R. Chin. On the detection of dominant points in digital curves. IEEE Trans. Pattern Anal. Mach. Intell. PAMI-11, pages 859-872, 1989.

10. E. Yamamoto et. al. Handwritten Kanji character recognition using the features extracted from multiple standpoints. In IEEE Conference on Pattern Recognition and Image Processing, 1981. 